\title{
Case Control Study
}

\section{Opioids and Risk of Type 2 Diabetes in Adults with Non-Cancer Pain}

Lin Li, MD, PhD'1, Soko Setoguchi, MD, DrPH ${ }^{2}$, Howard Cabral, $\mathrm{PhD}^{3}$, and Susan Jick, DSc ${ }^{1}$

From: 'Boston University School of Medicine, Boston, MA; ${ }^{2}$ Duke

Clinical Research Institute,

Durham, NC; and 'Boston University School of Public Health,

Boston, MA

Drs Li and Jick, Boston Collaborative Drug Surveillance Program, Boston University School of Medicine, Boston, MA.

Dr Setoguchi, Duke Clinical Research Institute, Durham, NC.

Dr Cabral, Department of

Blostatistics, Boston University School of Public Health, Boston,

MA.

Address Correspondence: Dr. Susan Jick 11 Muzzey Street Lexington, MA 02421

E-mail: Sjick@bu.edu

Disclaimer: There was no external funding in the preparation of this manuscript.

Conflict of interest: None.

Manuscript received: 03-22-2012 Revised manuscript received: o4-10-2012

Accepted for publication: o9-11-2012

Free full manuscript: www.painphysicianjournal.com
Background: While the use of opioids for chronic non-cancer pain (CNCP) has increased dramatically in the past 2 decades, concern exists about the safety of opioids, particularly with the extensive use among individuals with CNCP.

Objective: To assess the risk of type 2 diabetes (T2D) among adults exposed to opioids for non-cancer pain.

Study Design: Nested case-control study.

Setting: United Kingdom-based General Practice Research Database (GPRD).

Methods: Among 1.7 million opioid users with at least one prescription for an opioid to treat non-cancer pain in the GPRD (1990 - 2008), we identified all incident T2D cases with at least 2 years of medical history before their first diagnosis (index date). For each case we randomly selected up to 2 controls matched on age, gender, index date, and general practice. The same eligibility requirements were applied to controls as to cases. We defined "any exposure" as at least 2 prescriptions for an opioid within 2 years before the index date and defined "nonuse" as no use or only one prescription within 2 years (reference). For any exposure to opioids we further evaluated timing of use, cumulative use, and individual opioid type. Conditional logistic regression was used to estimate adjusted odds ratios (AORs) and 95\% confidence intervals (Cls) controlling for confounders.

Results: We identified 50,468 T2D cases to which we matched 100,415 controls. Cases were more likely than controls to be former smokers, heavier, and to have more comorbidities, co-medications, and visits to their general practitioners. After adjusting for important confounders there was no increased risk for T2D among those exposed to any opioid compared to nonusers ( $A O R=1.03,95 \% \mathrm{Cl} 1.00-1.06)$. The results did not change when we evaluated timing of use, cumulative use, or individual opioid type.

Limitations: Misclassification of exposure may have occurred; limited data for some individual opioid types.

Conclusion: This study found no association between use of opioids and risk of T2D among non-cancer adults.

Key words: Opioids, type 2 diabetes, non-cancer pain, General Practice Research Database GPRD, nested case-control, safety, adults

Pain Physician 2013; 16:77-88 
O pioid analgesics have been widely accepted for treatment of cancer-pain or acute severe pain (1-5). In the past 20 years the use of opioids for chronic non-cancer pain (CNCP) has increased dramatically in part due to improved awareness of the undertreatment of chronic pain and the introduction of new agents and/or new delivery formulations (6). Some adverse effects of opioid use are well known, such as nausea, constipation, sedation, and addition $(7,8)$, and recently, the Food and Drug Administration removed propoxyphene from the US market because this drug puts patients at risk for potentially serious or even fatal heart rhythm abnormalities (9). There are also other safety concerns surrounding this class of drugs such as hormonal and immune system effects where limited data exist on chronic or rare adverse effects, particularly in extensive users with CNCP (8).

Decreased plasma testosterone levels have long been documented in male narcotic addicts (10-12). More recently, studies have demonstrated similar hormonal effects for patients taking prescribed opioids for CNCP, which consistently found that the effect was not limited to testosterone, but also estrogen, luteinizing hormone, gonadotrophin releasing hormone, and dehydroepiandrosterone (13-19). These sex hormones are associated with adverse lipid profiles and insulin resistance (20). A number of studies have reported an association between low testosterone and type 2 diabetes (T2D), or suggested that low levels of testosterone are a precursory factor for the incidence of insulin resistance and T2D in healthy men (21-26). Post hoc analyses from 2 clinical trials found a significant reduction in the incidence of diabetes among women on hormone replacement therapy $(27,28)$.

Since opioids are known to increase the risk of hypogonadism $(8,29,30)$, we hypothesized that opioid use for non-cancer pain was associated with an increased risk of T2D among adults. To test this hypothesis we conducted a matched, nested case-control study using the United Kingdom (UK)-based General Practice Research Database (GPRD).

\section{Methods}

\section{Data Source and Study Sample}

Data were derived from the GPRD, a large primary care database that has been previously described in detail (31-33). Briefly, the GPRD is an ongoing longitudinal database that has collected data from over 350 general practices in the UK since 1987. It currently contains information about more than 3.5 million active patients, approximately $5 \%$ of the UK population, and has a representative distribution in terms of age and gender. The information recorded includes patient demographics and characteristics (e.g., height, weight, and smoking status), clinical diagnoses, drug prescriptions, consultant referrals, and hospitalizations. Validation studies have shown the high quality of recorded drug exposure and diagnoses (31-34).

The study sample comprised 1.7 million opioid users with at least one prescription for an opioid between January 1, 1990, and December 31, 2008, in the GPRD, who did not have a diagnosis of cancer (except non-melanoma skin cancer) any time before the firstrecorded opioid prescription. This study was reviewed and approved by the Independent Scientific Advisory Committee for UK Medicines and Healthcare Products Regulatory Agency database research.

\section{Case Selection}

From the study sample we identified all first-ever diagnosed T2D cases aged 18-80 years during 19902008. We required that each case have at least 2 years of medical history before their first-time recorded T2D diagnosis (i.e., index date). We excluded any patients with a diagnosis of cancer (except non-melanoma skin cancer), a history of use of hypoglycaemic agents, chronic pancreatitis, cystic fibrosis, haemochromatosis, Cushing syndrome, acromegaly, kidney or liver transplantation, human immunodeficiency virus/acquired immunodeficiency syndrome, or alcohol abuse any time before the index date, or those with acute pancreatitis within one year before the index date. We also excluded those with codes for other specified types of diabetes, e.g., type 1 diabetes, malnutritionrelated diabetes, steroid induced diabetes, and gestational diabetes, any time recorded in their medical history, or those with codes for pregnancy recorded within one year before, on, or one year after their index date.

To ensure high specificity for T2D diagnoses we required that each case be accompanied by follow-up information consistent with a diagnosis of diabetes (e.g., a prescription for an oral hypoglycaemic agent or insulin, treatment with diet, or referral to a diabetic clinic followed by progress notes from the clinic). In addition, we reviewed the full computer records of all patients younger than 35 years at diagnosis of diabetes without knowledge of exposure status, and excluded those who received insulin within the first 3 months of their diag- 
nosis since they were most likely to have type 1 diabetes $(35,36)$.

\section{Control Selection}

For each T2D case we randomly selected up to 2 controls with no diagnosis of diabetes, matched on age (the same year of birth), gender, index date, and general practice (GP) from the same study sample that generated the cases. The same inclusion and exclusion criteria applied to cases were applied to controls.

\section{Opioid Exposure}

We identified all opioid prescriptions recorded before the index date for all cases and controls from the computer records without knowledge of disease status. We determined the length of the closest prescription prior to the index date by dividing the prescribed quantity by its daily usage. If a patient was instructed to take the opioid "when required," we assumed that the patient took the opioid according to the maximum recommended daily dose to calculate prescription length.

We defined "any exposure" as at least 2 prescriptions for an opioid within 2 years before the index date. "Nonuse" was defined as no use of any opioids or only one prescription for an opioid recorded within 2 years before the index date (reference). "Any exposure" was further classified into "current use" if the most recent opioid prescription's supply ended within 90 days before the index date or after the index date. A person was classified as a "recent user" if their supply ended within 91-365 days prior to the index date, or "past user" if the prescription ended more than 365 days and less than 2 years prior to the index date. For "any exposure" we assessed and categorized the number of opioid prescriptions before the index date as 2-3, 4-10, 11-30, 31-50, 51-100, and $>100$ prescriptions. Prescription categories were defined to examine potential effect due to different lengths of cumulative use. Individual opioid type was categorized according to the last opioid prescribed before the index date. If different types of opioids were prescribed simultaneously for the last prescription, or if the supply of a second opioid overlapped with the last opioid prescription, they were classified as having use of opioid polytherapy.

We further grouped current users by the number of opioid prescriptions recorded before the index date $(2-3,4-10,11-30,31-50,51-100,>100)$ and by the last opioid type prescribed before the index date.

\section{Covariates}

We collected many covariates including lifestyle risk factors, co-morbidities, co-medications and types of pain, which are listed in Table 1.

\section{Statistical Analysis}

We conducted conditional logistic regression to estimate the unadjusted odds ratios (ORs) of T2D and $95 \%$ confidence intervals (Cls) among those with opioid exposure compared to those without while accounting for the matching factors. We evaluated each covariate for potential confounding by separately evaluating the associations with T2D and opioid exposure, and then examined the strength of potential confounding by assessing the effect of each confounder on the crude association between opioid exposure and T2D, one confounder at a time. We estimated the adjusted ORs of T2D and 95\% Cls after controlling for covariates which affected the crude association of opioid exposure and risk of T2D by $10 \%$ or more using conditional logistic regression, and adjusted ORs and $95 \% \mathrm{Cls}$ after controlling for all confounders that were associated with both opioid exposure and T2D. Finally, we conducted stratified analyses to examine whether the associations between each category of opioid exposure and T2D were homogeneous across gender, age (18-50, 51-60, 61-70, and 71-80 years), calendar year of the index date (1990-1994, 1995-1999, 2000-2004, and 2005-2008), and risk factors for developing T2D.

To examine whether the primary findings were robust we conducted 2 sensitivity analyses. First, to check the impact of potential misclassification of T2D, we repeated conditional logistic regression analyses among only drug-treated T2D cases and their matched controls. Second, given the potential misclassification of exposure due to as-needed prescribing, we re-defined opioid exposure based on the actual prescribed date of the last opioid prescription before the index date, not accounting for the duration, and checked the association between each category of opioid exposure and risk of T2D.

\section{Results}

We identified 50,468 eligible incident T2D cases from the study sample to which we matched 100,415 controls. Table 1 provides the distribution of baseline characteristics among the cases and controls. The T2D cases had a mean age of 61.5 years and $51.5 \%$ of cases were men. Age, gender, and calendar year of index 
Pain Physician: January/February 2013; 16:77-88

Table 1. Characteristics of cases and controls and risk of type 2 diabetes associated with covariates

\begin{tabular}{|c|c|c|c|}
\hline & Cases $\mathrm{N}=50,468(\%)$ & Controls N = 100,415 (\%) & Adjusted OR (95\% CI) * \\
\hline \multicolumn{4}{|l|}{ Age (years) } \\
\hline Mean \pm SD & $61.5 \pm 11.5$ & $61.5 \pm 11.5$ & $\mathrm{NA} \dagger$ \\
\hline \multicolumn{4}{|l|}{ Gender } \\
\hline Male & $25,972(51.5)$ & $51,613(51.4)$ & \multirow{2}{*}{$\mathrm{NA} \dagger$} \\
\hline Female & $24,496(48.5)$ & $48,802(48.6)$ & \\
\hline \multicolumn{4}{|c|}{ Calendar year of index date } \\
\hline $1990-1994$ & $4,320(8.56)$ & $8,563(8.53)$ & \multirow{4}{*}{$\mathrm{NA} \dagger$} \\
\hline $1995-1999$ & $11,190(22.2)$ & $22,269(22.2)$ & \\
\hline $2000-2004$ & $19,672(39.0)$ & $39,171(39.0)$ & \\
\hline $2005-2008$ & $15,286(30.3)$ & $30,412(30.3)$ & \\
\hline \multicolumn{4}{|l|}{ Smoking } \\
\hline Never & $23,174(45.9)$ & $48,263(48.1)$ & Reference \\
\hline Current & $9,728(19.3)$ & $21,625(21.5)$ & $1.13(1.10-1.17)$ \\
\hline Former & $13,103(26.0)$ & $20,038(20.0)$ & $1.23(1.19-1.27)$ \\
\hline Unknown & $4,463(8.84)$ & $10,489(10.4)$ & $1.29(1.22-1.36)$ \\
\hline \multicolumn{4}{|l|}{ BMI $\left(\mathrm{kg} / \mathrm{m}^{2}\right)$} \\
\hline$<18.5$ & $121(0.24)$ & $1,219(1.21)$ & $0.57(0.47-0.70)$ \\
\hline $18.5-24.9$ & $4,712(9.34)$ & $29,523(29.4)$ & Reference \\
\hline $25.0-29.9$ & $14,695(29.1)$ & $32,893(32.8)$ & $2.58(2.48-2.68)$ \\
\hline $30.0-39.9$ & $19,932(39.5)$ & $16,172(16.1)$ & $6.77(6.49-7.06)$ \\
\hline$\geq 40.0$ & $4,485(8.89)$ & $1,340(1.33)$ & $17.6(16.3-19.0)$ \\
\hline Unknown & $6,523(12.9)$ & $19,268(19.2)$ & $1.85(1.76-1.95)$ \\
\hline Drug abuse & $420(0.83)$ & $884(0.88)$ & $0.83(0.72-0.95)$ \\
\hline \multicolumn{4}{|c|}{ Number of GP visits, within 2 years before index date } \\
\hline Mean \pm SD & $32.5 \pm 23.2$ & $25.8 \pm 21.5$ & \\
\hline$<10$ & $6,954(13.8)$ & $25,187(25.1)$ & Reference \\
\hline $10-19$ & $8,803(17.4)$ & $20,740(20.7)$ & $1.20(1.15-1.25)$ \\
\hline $20-39$ & $19,191(38.0)$ & $33,249(33.1)$ & $1.25(1.20-1.30)$ \\
\hline $40-59$ & $10,320(20.5)$ & $14,551(14.5)$ & $1.27(1.21-1.34)$ \\
\hline$\geq 60$ & $5,200(10.3)$ & $6,688(6.66)$ & $1.19(1.11-1.27)$ \\
\hline \multicolumn{4}{|c|}{ Co-morbidities, any time before index date } \\
\hline Hyperlipidemia & $8,878(17.6)$ & $12,005(12.0)$ & $1.07(1.03-1.12)$ \\
\hline Hypertension & $24,590(48.7)$ & $28,777(28.7)$ & $1.40(1.35-1.45)$ \\
\hline Cardiovascular disease & $15,289(30.3)$ & $20,489(20.4)$ & $1.16(1.12-1.20)$ \\
\hline Asthma/COPD & $9,252(18.3)$ & $15,106(15.0)$ & $0.99(0.95-1.02)$ \\
\hline Osteoarthritis & $15,503(30.7)$ & $27,741(27.6)$ & $0.95(0.92-0.98)$ \\
\hline Autoimmune disease & $1,258(2.49)$ & $2,752(2.74)$ & $0.87(0.80-0.94)$ \\
\hline Fibromyalgia & $781(1.55)$ & $1,423(1.42)$ & $0.96(0.87-1.07)$ \\
\hline Epilepsy & $867(1.72)$ & $1,897(1.89)$ & $0.94(0.85-1.05)$ \\
\hline Parkinson disease & $167(0.33)$ & $527(0.52)$ & $0.66(0.54-0.80)$ \\
\hline Depression & $12,175(24.1)$ & $21,650(21.6)$ & $0.99(0.96-1.03)$ \\
\hline Schizophrenia & $538(1.07)$ & $685(0.68)$ & $1.38(1.20-1.59)$ \\
\hline Hyperthyroidism & $803(1.59)$ & $1,391(1.39)$ & $1.08(0.98-1.20)$ \\
\hline Psoriasis & $2,476(4.91)$ & $4,003(3.99)$ & $1.11(1.04-1.17)$ \\
\hline
\end{tabular}


Opioids and Risk of Type 2 Diabetes

Table 1 (cont.). Characteristics of cases and controls and risk of type 2 diabetes associated with covariates

\begin{tabular}{|c|c|c|c|}
\hline & Cases $N=50,468(\%)$ & Controls $N=100,415(\%)$ & Adjusted OR (95\% CI) * \\
\hline Chronic liver disease & $325(0.64)$ & $228(0.23)$ & $2.26(1.85-2.76)$ \\
\hline Chronic renal disease & $1,072(2.12)$ & $1,624(1.62)$ & $0.86(0.78-0.95)$ \\
\hline PCOS & $198(0.39)$ & $107(0.11)$ & $2.25(1.66-3.06)$ \\
\hline Surgery $\ddagger$ & $7,284(14.4)$ & $7,003(6.97)$ & $2.16(2.07-2.26)$ \\
\hline Injury $\ddagger$ & $781(1.55)$ & $1,471(1.46)$ & $1.03(0.93-1.14)$ \\
\hline \multicolumn{4}{|c|}{ Co-medications, within 90 days before index date } \\
\hline Lipid modifying therapy & $9,962(19.7)$ & $11,924(11.9)$ & $1.14(1.09-1.19)$ \\
\hline Antihypertensive drugs & $26,961(53.4)$ & $31,596(31.5)$ & $1.48(1.42-1.54)$ \\
\hline ASA & $12,087(24.0)$ & $14,673(14.6)$ & $1.13(1.08-1.17)$ \\
\hline Steroids & $7,079(14.0)$ & $8,561(8.53)$ & $1.46(1.39-1.53)$ \\
\hline NSAIDs & $7,999(15.9)$ & $13,518(13.5)$ & $0.95(0.92-0.99)$ \\
\hline Acetaminophen & $3,466(6.87)$ & $5,118(5.10)$ & $1.06(1.00-1.12)$ \\
\hline Antidepressants & $6,136(12.2)$ & $9,134(9.10)$ & $1.13(1.07-1.18)$ \\
\hline Antipsychotics & $1,960(3.88)$ & $2,483(2.47)$ & $1.34(1.25-1.45)$ \\
\hline Antiepileptics & $931(1.84)$ & $2,041(2.03)$ & $0.77(0.69-0.85)$ \\
\hline Oral contraceptives & $419(0.83)$ & $914(0.91)$ & $0.93(0.80-1.08)$ \\
\hline HRT & $1,293(2.56)$ & $3,377(3.36)$ & $0.72(0.67-0.78)$ \\
\hline \multicolumn{4}{|c|}{ Types of pain, within 90 days before index date } \\
\hline Neuralgia & $112(0.22)$ & $165(0.16)$ & $1.50(1.13-1.98)$ \\
\hline Headache & $724(1.43)$ & $1,001(1.00)$ & $1.27(1.13-1.42)$ \\
\hline Abdominal \& pelvic pain & $1,824(3.61)$ & $2,198(2.19)$ & $1.53(1.42-1.65)$ \\
\hline Musculoskeletal pain & $4,957(9.82)$ & $8,089(8.06)$ & $1.10(1.05-1.15)$ \\
\hline Chest pain & $1,318(2.61)$ & $1,284(1.28)$ & $1.72(1.57-1.88)$ \\
\hline Other pain & $1,056(2.09)$ & $1,648(1.64)$ & $1.09(0.99-1.19)$ \\
\hline
\end{tabular}

ASA- acetylsalicylic acid, BMI- body mass index, CI-confidence interval, COPD-chronic obstructive pulmonary disease, GP-general practice, HRT-hormone replacement therapy, NSAIDs- nonsteroidal anti-inflammatory drugs, OR-odds ratio, PCOS-polycystic ovary syndrome, SDstandard deviation

* Estimates were obtained from a conditional logistic regression model including all covariates listed in this table.

$\dagger$ Matching variable.

¥ Assessed within 90 days before the index date.

date were similar among cases and controls because of matching. Cases and controls had a similar distribution of length of medical history recorded before the index date with an average of 9 years. Cases were more likely than controls to be former smokers ( $26.0 \%$ vs. $20.0 \%)$, though controls were slightly more likely to be current smokers. Cases were also more likely to have a body mass index (BMI) $\geq 30 \mathrm{~kg} / \mathrm{m}^{2}(48.4 \%$ vs. $17.4 \%)$ and more GP visits within 2 years before the index date than controls (mean 33 vs. 26 visits). Compared to controls T2D cases generally had more co-morbidities, such as hyperlipidemia and cardiovascular disease (CVD), and took more co-medications, such as lipid-modifying therapy, antihypertensive drugs, steroids, non-opioid analge- sics, antidepressants, and antipsychotics. Cases also had more surgeries and suffered slightly more from various types of pain within 90 days before the index date compared to controls.

A total of 20,847 T2D cases $(41.3 \%)$ and 33,830 controls $(33.7 \%)$ had received at least 2 opioid prescriptions within 2 years before their index date. Of those, $11,524(22.8 \%)$ cases and $17,518(17.5 \%)$ controls were current users, i.e., used opioids within 90 days before the index date, $11,191(22.2 \%)$ cases and $16,454(16.4 \%)$ controls had more than 10 opioid prescriptions. Codeine, dihydrocodeine, propoxyphene, and tramadol were the most commonly prescribed opioids with a slightly higher proportion of each in 
cases compared to controls. The unadjusted OR for T2D was $1.41(95 \% \mathrm{Cl} ; 1.38-1.45)$ for users of any opioid compared with no opioid exposure. However, adjustment for BMI, number of GP visits, and antihypertensive drug use removed the association of opioid exposure with T2D (adjusted OR $=1.03,95 \% \mathrm{Cl}$; 1.00-1.06). Nor was there an effect when we evaluated timing of opioid exposure, cumulative opioid use, and individual opioid types after these adjustments.
The results did not change when we simultaneously adjusted for all covariates (including lifestyle risk factors, co-morbidities, co-medications, and types of pain) which were associated with both opioid exposure and T2D (Table 2).

For current opioid users we further examined cumulative use and individual opioid types in relation to the risk of T2D (Table 3). Although each category of cumulative use was associated with an increased risk of T2D

Table 2. Odds ratios and 95\% confidence intervals for opioid exposures in relation to risk of type 2 diabetes

\begin{tabular}{|c|c|c|c|c|c|}
\hline & $\begin{array}{c}\text { Cases } \\
\mathbf{N}=\mathbf{5 0 , 4 6 8}(\%)\end{array}$ & $\begin{array}{c}\text { Controls } \\
\mathrm{N}=\mathbf{1 0 0 , 4 1 5}(\%)\end{array}$ & $\begin{array}{c}\text { OR* } \\
(95 \% \mathrm{CI})\end{array}$ & $\begin{array}{l}\text { Multivariate } \\
\text { Adjusted OR† } \\
\text { (95\% CI) }\end{array}$ & $\begin{array}{c}\text { Multivariate } \\
\text { Adjusted OR } \\
\text { (95\% CI) }\end{array}$ \\
\hline Nonuse & $29,621(58.7)$ & $66,585(66.3)$ & Reference & Reference & Reference \\
\hline Any opioid use & $20,847(41.3)$ & $33,830(33.7)$ & $1.41(1.38-1.45)$ & $1.03(1.00-1.06)$ & $1.03(1.00-1.06)$ \\
\hline \multicolumn{6}{|l|}{ Timing of use } \\
\hline Current use & $11,524(22.8)$ & $17,518(17.5)$ & $1.52(1.48-1.56)$ & $1.02(0.98-1.05)$ & $1.00(0.96-1.04)$ \\
\hline Recent use & $5,529(11.0)$ & $9,423(9.38)$ & $1.34(1.29-1.39)$ & $1.04(1.00-1.09)$ & $1.05(1.01-1.10)$ \\
\hline Past use & $3,794(7.52)$ & $6,889(6.86)$ & $1.26(1.20-1.31)$ & $1.06(1.01-1.11)$ & $1.06(1.01-1.12)$ \\
\hline \multicolumn{6}{|c|}{ Cumulative use, number of prescriptions } \\
\hline $2-3$ & $4,126(8.18)$ & $7,692(7.66)$ & $1.21(1.16-1.26)$ & $1.05(1.01-1.10)$ & $1.04(0.99-1.09)$ \\
\hline $4-10$ & $5,530(11.0)$ & $9,684(9.64)$ & $1.31(1.26-1.36)$ & $1.02(0.98-1.07)$ & $1.01(0.97-1.05)$ \\
\hline $11-30$ & $5,087(10.1)$ & $7,656(7.62)$ & $1.54(1.48-1.60)$ & $1.07(1.02-1.12)$ & $1.07(1.02-1.12)$ \\
\hline $31-50$ & $2,124(4.21)$ & $3,294(3.28)$ & $1.50(1.42-1.59)$ & $0.97(0.90-1.03)$ & $0.98(0.91-1.05)$ \\
\hline $51-100$ & $2,427(4.81)$ & $3,410(3.40)$ & $1.67(1.58-1.77)$ & $0.99(0.93-1.06)$ & $1.01(0.94-1.08)$ \\
\hline$>100$ & $1,553(3.08)$ & $2,094(2.09)$ & $1.76(1.64-1.88)$ & $1.00(0.92-1.08)$ & $1.03(0.94-1.12)$ \\
\hline \multicolumn{6}{|l|}{ Opioid type } \\
\hline Morphine & $116(0.23)$ & $162(0.16)$ & $1.65(1.29-2.09)$ & $1.18(0.89-1.55)$ & $1.10(0.82-1.46)$ \\
\hline Oxycodone & $27(0.05)$ & $31(0.03)$ & $1.97(1.17-3.30)$ & $1.29(0.70-2.39)$ & $1.24(0.66-2.33)$ \\
\hline Dihydrocodeine & $4,822(9.55)$ & $7,560(7.53)$ & $1.46(1.41-1.52)$ & $1.08(1.03-1.13)$ & $1.08(1.03-1.13)$ \\
\hline Diamorphine & $11(0.02)$ & $8(0.01)$ & $3.08(1.24-7.66)$ & $2.62(0.88-7.83)$ & $1.73(0.58-5.16)$ \\
\hline Codeine & $8,953(17.7)$ & $14,728(14.7)$ & $1.40(1.36-1.44)$ & $1.03(1.00-1.07)$ & $1.02(0.99-1.06)$ \\
\hline Meperidine & $33(0.07)$ & $52(0.05)$ & $1.41(0.91-2.18)$ & $1.25(0.76-2.07)$ & $1.13(0.68-1.88)$ \\
\hline Fentanyl & $18(0.04)$ & $37(0.04)$ & $1.12(0.64-1.98)$ & $0.67(0.35-1.27)$ & $0.64(0.33-1.21)$ \\
\hline Propoxyphene & $4,678(9.27)$ & $8,018(7.98)$ & $1.32(1.27-1.38)$ & $0.98(0.94-1.03)$ & $0.99(0.94-1.03)$ \\
\hline Buprenorphine & $68(0.13)$ & $115(0.11)$ & $1.36(1.00-1.84)$ & $0.89(0.63-1.26)$ & $0.90(0.64-1.29)$ \\
\hline Tramadol & $1,240(2.46)$ & $1,864(1.86)$ & $1.54(1.43-1.66)$ & $1.01(0.92-1.10)$ & $1.02(0.93-1.11)$ \\
\hline Meptazinol & $43(0.09)$ & $57(0.06)$ & $1.76(1.18-2.61)$ & $1.22(0.78-1.91)$ & $1.12(0.71-1.78)$ \\
\hline Polytherapy & $811(1.61)$ & $1,129(1.12)$ & $1.65(1.50-1.81)$ & $1.09(0.98-1.21)$ & $1.05(0.94-1.17)$ \\
\hline Others & $27(0.05)$ & $69(0.07)$ & $0.87(0.56-1.36)$ & $0.79(0.47-1.32)$ & $0.94(0.56-1.58)$ \\
\hline
\end{tabular}

CI- confidence interval, OR-odds ratio

* Adjusted for matching variables.

$\dagger$ Adjusted for body mass index, number of general practice visits, and use of antihypertensive drugs which changed the OR by $10 \%$ or more in addition to matching variables.

¥ Adjusted for smoking, body mass index, history of drug abuse, number of general practice visits, co-morbidities (including hyperlipidemia, hypertension, cardiovascular disease, osteoarthritis, autoimmune disease, Parkinson disease, schizophrenia, psoriasis, chronic liver disease, chronic renal disease, and surgery), all co-medications except oral contraceptives, and all types of pain except other pain in addition to matching variables. 
when only controlling for the matching factors, all ORs decreased to the null after adjustment for confounders. There was no effect for any individual opioid type.

Stratification by gender did not yield material differences in diabetes risk (Table 4). Nor were there any material differences when we stratified on age, calendar year of index date, or risk factors for T2D including BMI $\left(<25, \geq 25 \mathrm{~kg} / \mathrm{m}^{2}\right)$, hypertension, dyslipidemia, and history of CVD (data not shown).
Among the 50,468 T2D cases, 39,557 (78.4\%) had been prescribed at least one prescription for a hypoglycemic agent. When we repeated the analyses among the drug-treated T2D cases and their matched controls there were few changes in the effect measures between each category of opioid exposure and risk of T2D compared to those among the whole study subjects (Table $5)$. When we redefined opioid exposure based on the actual prescription dates of opioids, we found results

Table 3. Odds ratios and 95\% confidence intervals for current opioid exposure combined with duration of use and opioid type in relation to risk of type 2 diabetes.

\begin{tabular}{|c|c|c|c|c|c|}
\hline & $\begin{array}{c}\text { Cases } \\
\mathbf{N}=\mathbf{5 0 , 4 6 8}(\%)\end{array}$ & $\begin{array}{c}\text { Controls } \\
\mathrm{N}=\mathbf{1 0 0 , 4 1 5}(\%)\end{array}$ & $\begin{array}{c}\text { OR* } \\
(95 \% \mathrm{CI})\end{array}$ & $\begin{array}{c}\text { Multivariate Adjusted } \\
\text { OR } \dagger \\
(95 \% \text { CI }) \\
\end{array}$ & $\begin{array}{c}\text { Multivariate Adjusted } \\
\text { OR } \% \\
(95 \% \text { CI }) \\
\end{array}$ \\
\hline Nonuse & $29,621(58.7)$ & $66,585(66.3)$ & Reference & Reference & Reference \\
\hline \multicolumn{6}{|c|}{ Current use, number of prescriptions } \\
\hline $2-3$ & $1,055(2.09)$ & $1,738(1.73)$ & $1.37(1.27-1.48)$ & $1.19(1.09-1.30)$ & $1.08(0.98-1.18)$ \\
\hline $4-10$ & $2,076(4.11)$ & $3,321(3.31)$ & $1.43(1.35-1.51)$ & $1.08(1.01-1.16)$ & $1.03(0.96-1.10)$ \\
\hline $11-30$ & $3,142(6.23)$ & $4,810(4.79)$ & $1.50(1.43-1.58)$ & $1.00(0.95-1.06)$ & $0.99(0.94-1.05)$ \\
\hline $31-50$ & $1,657(3.28)$ & $2,651(2.64)$ & $1.45(1.36-1.55)$ & $0.92(0.85-0.99)$ & $0.93(0.86-1.00)$ \\
\hline $51-100$ & $2,154(4.27)$ & $3,026(3.01)$ & $1.66(1.57-1.76)$ & $0.97(0.91-1.04)$ & $0.99(0.92-1.07)$ \\
\hline$>100$ & $1,440(2.85)$ & $1,972(1.96)$ & $1.72(1.60-1.84)$ & $0.96(0.88-1.04)$ & $0.99(0.90-1.08)$ \\
\hline Recent use & $5,529(11.0)$ & $9,423(9.38)$ & $1.34(1.29-1.39)$ & $1.04(0.99-1.08)$ & $1.05(1.00-1.10)$ \\
\hline Past use & $3,794(7.52)$ & $6,889(6.86)$ & $1.26(1.21-1.31)$ & $1.05(1.00-1.11)$ & $1.06(1.01-1.12)$ \\
\hline \multicolumn{6}{|c|}{ Current use, opioid type } \\
\hline Morphine & $91(0.18)$ & $122(0.12)$ & $1.73(1.32-2.28)$ & $1.21(0.88-1.65)$ & $1.09(0.79-1.51)$ \\
\hline Oxycodone & $25(0.05)$ & $26(0.03)$ & $2.18(1.25-3.78)$ & $1.32(0.69-2.51)$ & $1.28(0.66-2.48)$ \\
\hline Dihydrocodeine & $2,626(5.20)$ & $3,745(3.73)$ & $1.62(1.53-1.70)$ & $1.09(1.02-1.16)$ & $1.07(1.01-1.14)$ \\
\hline Diamorphine & $4(0.01)$ & $2(0.00)$ & $4.51(0.82-24.7)$ & $2.08(0.27-15.9)$ & $1.01(0.13-7.91)$ \\
\hline Codeine & $4,841(9.59)$ & $7,254(7.22)$ & $1.55(1.49-1.62)$ & $1.05(1.00-1.10)$ & $1.03(0.98-1.08)$ \\
\hline Meperidine & $17(0.03)$ & $20(0.02)$ & $1.89(0.99-3.62)$ & $1.57(0.73-3.36)$ & $1.19(0.55-2.56)$ \\
\hline Fentanyl & $14(0.03)$ & $29(0.03)$ & $1.10(0.58-2.08)$ & $0.67(0.32-1.38)$ & $0.66(0.32-1.37)$ \\
\hline Propoxyphene & $2,546(5.04)$ & $4,406(4.39)$ & $1.32(1.25-1.39)$ & $0.90(0.85-0.95)$ & $0.90(0.85-0.96)$ \\
\hline Buprenorphine & $54(0.11)$ & $80(0.08)$ & $1.58(1.12-2.23)$ & $0.99(0.66-1.48)$ & $0.97(0.65-1.46)$ \\
\hline Tramadol & $726(1.44)$ & $1,055(1.05)$ & $1.60(1.45-1.76)$ & $0.98(0.88-1.10)$ & $0.97(0.86-1.09)$ \\
\hline Meptazinol & $19(0.04)$ & $24(0.02)$ & $1.86(1.02-3.40)$ & $1.34(0.67-2.66)$ & $1.26(0.62-2.58)$ \\
\hline Polytherapy & $543(1.08)$ & $709(0.71)$ & $1.77(1.58-1.98)$ & $1.06(0.93-1.21)$ & $0.99(0.86-1.13)$ \\
\hline Others & $18(0.04)$ & $46(0.05)$ & $0.87(0.50-1.49)$ & $0.73(0.38-1.37)$ & $0.90(0.47-1.71)$ \\
\hline Recent use & $5,529(11.0)$ & $9,423(9.38)$ & $1.34(1.29-1.39)$ & $1.04(1.00-1.09)$ & $1.05(1.01-1.10)$ \\
\hline Past use & $3,794(7.52)$ & $6,889(6.86)$ & $1.26(1.21-1.31)$ & $1.06(1.01-1.11)$ & $1.06(1.01-1.12)$ \\
\hline
\end{tabular}

CI - confidence interval, OR - odds ratio

* Adjusted for matching variables.

$\dagger$ Adjusted for body mass index, number of general practice visits, and use of antihypertensive drugs which changed the OR by $10 \%$ or more in addition to matching variables.

¥ Adjusted for smoking, body mass index, history of drug abuse, number of general practice visits, co-morbidities (including hyperlipidemia, hypertension, cardiovascular disease, osteoarthritis, autoimmune disease, Parkinson disease, schizophrenia, psoriasis, chronic liver disease, chronic renal disease, and surgery), all co-medications except oral contraceptives, and all types of pain except other pain in addition to matching variables. 
Pain Physician: January/February 2013; 16:77-88

Table 4. Odds ratios and 95\% confidence intervals for opioid exposures in relation to risk of type 2 diabetes stratified on gender.

\begin{tabular}{|c|c|c|c|c|c|c|}
\hline & \multicolumn{3}{|c|}{ Male } & \multicolumn{3}{|c|}{ Female } \\
\hline & $\begin{array}{c}\text { Cases } \\
\mathbf{N}=\mathbf{2 5 , 9 7 2}(\%)\end{array}$ & $\begin{array}{c}\text { Controls } \\
\mathbf{N}=\mathbf{5 1 , 6 1 3}(\%)\end{array}$ & $\begin{array}{l}\text { Multivariate } \\
\text { Adjusted OR* } \\
\text { (95\% CI) }\end{array}$ & $\begin{array}{c}\text { Cases } \\
\mathrm{N}=\mathbf{2 4 , 4 9 6} \\
(\%)\end{array}$ & $\begin{array}{c}\text { Controls } \\
\text { N }=48,802 \\
(\%)\end{array}$ & $\begin{array}{l}\text { Multivariate } \\
\text { Adjusted OR* } \\
\text { (95\% CI) }\end{array}$ \\
\hline Nonuse & $16,705(64.3)$ & $36,200(70.1)$ & Reference & $12,916(52.7)$ & $30,385(62.3)$ & Reference \\
\hline Any opioid use & $9,267(35.7)$ & $15,413(29.9)$ & $0.98(0.94-1.02)$ & $11,580(47.3)$ & $18,417(37.7)$ & $1.08(1.03-1.12)$ \\
\hline \multicolumn{7}{|l|}{ Timing of use } \\
\hline Current use & $4,977(19.2)$ & $7,618(14.8)$ & $0.98(0.93-1.03)$ & $6,547(26.7)$ & $9,900(20.3)$ & $1.02(0.97-1.07)$ \\
\hline Recent use & $2,493(9.60)$ & $4,423(8.57)$ & $0.98(0.92-1.04)$ & $3,036(12.4)$ & $5,000(10.3)$ & $1.13(1.06-1.20)$ \\
\hline Past use & $1,797(6.92)$ & $3,372(6.53)$ & $0.99(0.93-1.07)$ & $1,997(8.15)$ & $3,517(7.21)$ & $1.15(1.07-1.23)$ \\
\hline \multicolumn{7}{|c|}{ Cumulative use, number of prescriptions } \\
\hline $2-3$ & $2,104(8.10)$ & $3,914(7.58)$ & $0.99(0.93-1.06)$ & $2,022(8.25)$ & $3,778(7.74)$ & $1.10(1.02-1.18)$ \\
\hline $4-10$ & $2,622(10.1)$ & $4,524(8.77)$ & $0.96(0.90-1.02)$ & $2,908(11.9)$ & $5,160(10.6)$ & $1.07(1.01-1.14)$ \\
\hline $11-30$ & $2,162(8.32)$ & $3,357(6.50)$ & $1.03(0.96-1.10)$ & $2,925(11.9)$ & $4,299(8.81)$ & $1.11(1.04-1.18)$ \\
\hline $31-50$ & $884(3.40)$ & $1,385(2.68)$ & $0.95(0.86-1.05)$ & $1,240(5.06)$ & $1,909(3.91)$ & $1.00(0.91-1.10)$ \\
\hline $51-100$ & $938(3.61)$ & $1,423(2.76)$ & $0.97(0.87-1.07)$ & $1,489(6.08)$ & $1,987(4.07)$ & $1.04(0.95-1.14)$ \\
\hline$>100$ & $557(2.14)$ & $810(1.57)$ & $1.00(0.87-1.14)$ & $996(4.07)$ & $1,284(2.63)$ & $1.05(0.94-1.17)$ \\
\hline \multicolumn{7}{|l|}{ Opioid type } \\
\hline Morphine & $55(0.21)$ & $78(0.15)$ & $1.02(0.68-1.53)$ & $61(0.25)$ & $84(0.17)$ & $1.18(0.79-1.77)$ \\
\hline Oxycodone & $14(0.05)$ & $13(0.03)$ & $1.82(0.73-4.57)$ & $13(0.05)$ & $18(0.04)$ & $0.82(0.34-1.99)$ \\
\hline Dihydrocodeine & $2,225(8.57)$ & $3,625(7.02)$ & $1.01(0.94-1.08)$ & $2,597(10.6)$ & $3,935(8.06)$ & $1.16(1.08-1.24)$ \\
\hline Diamorphine & $8(0.03)$ & $6(0.01)$ & $0.98(0.28-3.38)$ & $3(0.01)$ & $2(0.00)$ & NA \\
\hline Codeine & $3,900(15.0)$ & $6,579(12.8)$ & $0.98(0.93-1.03)$ & $5,053(20.6)$ & $8,149(16.7)$ & $1.07(1.02-1.13)$ \\
\hline Meperidine & $18(0.07)$ & $30(0.06)$ & $0.93(0.47-1.83)$ & $15(0.06)$ & $22(0.05)$ & $1.54(0.71-3.34)$ \\
\hline Fentanyl & $5(0.02)$ & $20(0.04)$ & $0.30(0.10-0.92)$ & $13(0.05)$ & $17(0.03)$ & $1.10(0.48-2.52)$ \\
\hline Propoxyphene & $2,058(7.92)$ & $3,474(6.73)$ & $0.98(0.91-1.05)$ & $2,620(10.7)$ & $4,544(9.31)$ & $1.00(0.94-1.07)$ \\
\hline Buprenorphine & $41(0.16)$ & $62(0.12)$ & $1.12(0.71-1.78)$ & $27(0.11)$ & $53(0.11)$ & $0.68(0.40-1.18)$ \\
\hline Tramadol & $570(2.19)$ & $912(1.77)$ & $0.95(0.84-1.08)$ & $670(2.74)$ & $952(1.95)$ & $1.09(0.96-1.23)$ \\
\hline Meptazinol & $17(0.07)$ & $29(0.06)$ & $0.81(0.41-1.60)$ & $26(0.11)$ & $28(0.06)$ & $1.49(0.78-2.85)$ \\
\hline Polytherapy & $340(1.31)$ & $541(1.05)$ & $0.90(0.76-1.06)$ & $471(1.92)$ & $588(1.20)$ & $1.19(1.02-1.38)$ \\
\hline Others & $16(0.06)$ & $44(0.09)$ & $0.93(0.48-1.82)$ & $11(0.04)$ & $25(0.05)$ & $0.98(0.41-2.30)$ \\
\hline
\end{tabular}

CI-confidence interval, OR- odds ratio

* Adjusted for smoking, body mass index, history of drug abuse, number of general practice visits, co-morbidities (including hyperlipidemia, hypertension, cardiovascular disease, osteoarthritis, autoimmune disease, Parkinson disease, schizophrenia, psoriasis, chronic liver disease, chronic renal disease, and surgery), all co-medications except oral contraceptives, and all types of pain except other pain in addition to matching variables.

similar to those when we defined exposure taking into account the duration of last available opioid prescription before the index date (data not shown).

\section{Discussion}

In the current study in general UK population, although any exposure to opioids was associated with an increased risk of T2D when only adjusting for the matching factors, there was no effect of opioids on T2D after further adjusting for BMI, number of GP visits, and concomitant antihypertensive drug use. Nor was there an effect when we evaluated timing of opioid exposure, cumulative opioid use, and individual opioid type after adjustments. BMI, hypertension, concomitant steroid use, and antipsychotics use were independently associated with an increased risk of T2D, findings that are consistent with prior knowledge of diabetes epidemiology (37-39). 
Table 5. Odds ratios and 95\% confidence intervals for opioid exposures in relation to risk of type 2 diabetes among drug-treated cases and controls.

\begin{tabular}{|c|c|c|c|c|}
\hline & $\begin{array}{c}\text { Cases } \\
\mathrm{N}=\mathbf{3 9 , 5 5 7}(\%) \\
\end{array}$ & $\begin{array}{c}\text { Controls } \\
\mathrm{N}=\mathbf{7 8 , 6 9 4}(\%)\end{array}$ & $\begin{array}{c}\mathbf{O R}^{*} \\
(95 \% \mathrm{CI}) \\
\end{array}$ & $\begin{array}{c}\text { Multivariate Adjusted OR } \\
(95 \% \text { CI) }\end{array}$ \\
\hline Nonuse & $23,449(59.3)$ & $52,994(67.3)$ & Reference & Reference \\
\hline Any opioid use & $16,108(40.7)$ & $25,700(32.7)$ & $1.45(1.41-1.48)$ & $1.06(1.02-1.09)$ \\
\hline \multicolumn{5}{|l|}{ Timing of use } \\
\hline Current use & $8,842(22.4)$ & $13,139(16.7)$ & $1.56(1.51-1.61)$ & $1.03(0.99-1.07)$ \\
\hline Recent use & $4,299(10.9)$ & $7,284(9.26)$ & $1.36(1.30-1.42)$ & $1.06(1.01-1.12)$ \\
\hline Past use & $2,967(7.50)$ & $5,277(6.71)$ & $1.29(1.23-1.36)$ & $1.10(1.04-1.17)$ \\
\hline \multicolumn{5}{|c|}{ Cumulative use, number of prescriptions } \\
\hline $2-3$ & $3,240(8.19)$ & $6,078(7.72)$ & $1.21(1.16-1.27)$ & $1.05(0.99-1.10)$ \\
\hline $4-10$ & $4,285(10.8)$ & $7,467(9.49)$ & $1.33(1.27-1.38)$ & $1.03(0.98-1.08)$ \\
\hline $11-30$ & $3,942(9.97)$ & $5,833(7.41)$ & $1.58(1.51-1.65)$ & $1.09(1.03-1.15)$ \\
\hline $31-50$ & $1,635(4.13)$ & $2,403(3.05)$ & $1.60(1.50-1.71)$ & $1.04(0.96-1.13)$ \\
\hline $51-100$ & $1,864(4.71)$ & $2,458(3.12)$ & $1.81(1.69-1.92)$ & $1.08(1.00-1.17)$ \\
\hline$>100$ & $1,142(2.89)$ & $1,461(1.86)$ & $1.88(1.73-2.03)$ & $1.09(0.98-1.20)$ \\
\hline \multicolumn{5}{|l|}{ Opioid type } \\
\hline Morphine & $89(0.22)$ & $117(0.15)$ & $1.76(1.33-2.33)$ & $1.15(0.83-1.61)$ \\
\hline Oxycodone & $20(0.05)$ & $21(0.03)$ & $2.18(1.18-4.04)$ & $1.38(0.65-2.94)$ \\
\hline Dihydrocodeine & $3,718(9.40)$ & $5,700(7.24)$ & $1.51(1.44-1.58)$ & $1.12(1.06-1.19)$ \\
\hline Diamorphine & $8(0.02)$ & $6(0.01)$ & $3.00(1.04-8.68)$ & $1.99(0.53-7.54)$ \\
\hline Codeine & $6,751(17.1)$ & $10,970(13.9)$ & $1.43(1.38-1.48)$ & $1.04(1.00-1.09)$ \\
\hline Meperidine & $28(0.07)$ & $38(0.05)$ & $1.64(1.00-2.67)$ & $1.20(0.68-2.13)$ \\
\hline Fentanyl & $12(0.03)$ & $28(0.04)$ & $0.99(0.50-1.94)$ & $0.54(0.24-1.18)$ \\
\hline Propoxyphene & $3,826(9.67)$ & $6,445(8.19)$ & $1.36(1.30-1.42)$ & $1.02(0.96-1.07)$ \\
\hline Buprenorphine & $52(0.13)$ & $86(0.11)$ & $1.39(0.98-1.96)$ & $0.95(0.63-1.44)$ \\
\hline Tramadol & $908(2.30)$ & $1,321(1.68)$ & $1.60(1.47-1.75)$ & $1.05(0.94-1.17)$ \\
\hline Meptazinol & $32(0.08)$ & $44(0.06)$ & $1.72(1.09-2.71)$ & $1.27(0.75-2.16)$ \\
\hline Polytherapy & $640(1.62)$ & $865(1.10)$ & $1.71(1.54-1.90)$ & $1.09(0.96-1.23)$ \\
\hline Others & $24(0.06)$ & $59(0.07)$ & $0.91(0.57-1.47)$ & $0.98(0.56-1.70)$ \\
\hline
\end{tabular}

CI-confidence interval, OR-odds ratio

* Adjusted for matching variables.

$\dagger$ Adjusted for smoking, body mass index, history of drug abuse, number of general practice visits, co-morbidities (including hyperlipidemia, hypertension, cardiovascular disease, osteoarthritis, autoimmune disease, Parkinson disease, schizophrenia, psoriasis, chronic liver disease, chronic renal disease, and surgery), all co-medications except oral contraceptives, and all types of pain except other pain in addition to matching variables.

A number of studies have reported an association between low levels of testosterone and insulin resistance, impaired glucose tolerance, or T2D in men $(21-26,40,41)$. In a prospective cohort of the Massachusetts Male Aging Study, Stellato and colleagues (25) found that low levels of mean baseline free testosterone were associated with the risk of developing diabetes. Interestingly, high not low testosterone levels are associated with higher risk of T2D in women $(22,41)$, which was supported by the ob- servation of insulin resistance in women with polycystic ovary syndrome and in non-diabetic women with abdominal obesity $(20,42)$. In addition, both estrogen deficiency and estrogen excess are believed to be associated with deterioration in insulin resistance, but the former is linked with deterioration in glucose homeostasis and the latter with glucose tolerance (43). However, to date we found only one study that reported directly on opioid use in relation to risk of T2D. This paper reported that current 
opioid use was associated with a 1.6-fold increased risk of diabetes compared to nonuse among women at risk or infected with human immunodeficiency virus (44). Given the highly selected study population, direct comparison of results is not possible. It should also be noted that use of highly active antiretroviral therapy has itself been associated with insulin resistance or diabetes (45).

Our study found that T2D cases were more likely to have the well-established diabetes risk factors, such as CVD, hypertension, and a high BMI, factors that were also associated with opioid exposure. Moreover, T2D cases were more likely to have GP visits than controls, suggesting the possibility that T2D cases have more opportunities to be screened for diabetes than controls. To control for this possible detection bias we adjusted for the number of GP visits occurring within 2 years before the index date, and found the crude OR fell from 1.52 to 1.11 for current opioid use. After we further controlled for BMI and antihypertensive drug use, all ORs for each category of opioid exposure decreased to around one. When we further adjusted for co-morbidities, co-medications, and types of pain associated with both opioid exposure and T2D, there were no material changes in the effect measures. These findings suggest that the crude positive associations were attributed to a combination of detection bias, poorer health in opioid users in contrast with nonusers, and confounding.

T2D does not have an acute clinical onset and it is likely that some cases had the illness for some time prior to their diagnosis. This could have resulted in some misclassification of exposure. The evaluation of different length of cumulative use should provide insight into the effects of this potential misclassification, as those with the longest exposure would be most likely to be truly exposed prior to disease onset and, if there were a true positive effect, it would be strongest with the longest use. We did not see evidence of a duration effect in these data so this is not a likely explanation for our results. In addition, some opioid prescriptions had ambiguous instructions for use, which could also have resulted in some misclassification of exposure when we defined timing of use based on the calculated duration. Nevertheless, when we redefined exposure based on the actual date of receipt of the last opioid prescription before the index date, not accounting for the length of the last prescription, we still did not find any association between opioid use and risk of T2D. Codeine in combination with paracetamol or ibuprofen and dihydrocodeine in combination with paracetamol, which are used for the treatment of mild to moderate pain, are available over-the-counter (OTC) in the UK, so it is possible that we missed some use of these OTC drugs in this study. However, this is unlikely to have had much impact on extensive users because people who receive more than minimal analgesia most often get prescriptions from their GPs to avoid paying out of pocket. Moreover, most opioids are prescription drugs and there are no effects for any individual opioid type in this study, so our results are unlikely to be explained by this kind of misclassification.

It is also possible that people who become prescription opioid abusers or addicts seek illegal sources for additional opioids which are not recorded in the GPRD. In our study we found no more than $1 \%$ of study subjects had a recorded history of drug abuse which is a strong predictor of opioid misuse in chronic pain patients (46). After we excluded these subjects, although they likely under represent the total number of prescription opioid abusers, we did not find any changes in our primary findings. In addition, if misclassification of exposure due to illegal opioid usage existed in our study, it would be more likely to occur in young adults than elderly adults, because young age is another predictor of opioid misuse in chronic pain patients (46). However, stratification by age did not yield material differences in diabetes risk.

In this study, we identified T2D cases based on computer-recorded diagnosis codes, rather than fasting glucose measurement. Although we required each case diagnosis to be accompanied by follow-up information consistent with a diagnosis of diabetes, we cannot rule out the possibility of case misclassification. However, when we repeated the analysis restricted to drug-treated T2D cases, who were unlikely to be false positive cases (47), and their matched controls, we found results similar to those among all study subjects indicating that our results are unlikely to be attributable to misclassification of disease.

In order to capture the maximum possible information on extensive (long-term) opioid use, we included both new and prevalent users in our study. To examine the impact of including people with unknown duration of use (prevalent use) in the study sample, we repeated the analysis restricted to new opioid users who had at least a one year washout period before their first recorded opioid prescription. The results were not materially different than the results in all study subjects.

In this study we did not control for family history of diabetes or physical activity level, because these data are not available in the GPRD. Nevertheless, our 
findings are not likely to be confounded by these risk factors. Although family history of diabetes is an independent risk factor for diabetes, it is unlikely to be associated with opioid use. Low physical activity level is another risk factor for diabetes. Since opioid users generally suffer from more co-morbidities and pain symptoms, they are less likely to have regular exercise than nonusers. In this scenario, we would predict an increased risk for opioid users because of confounding by physical activity level, so missing information on these covariates would not explain our finding.

\section{Conclusion}

Our results do not support the hypothesis that opioid treatment is associated with an increased risk of
T2D among non-cancer adults. In light of the increasing use of opioids in patients with chronic pain, which can undermine overall physical, psychological, and social well-being of the affected individuals, these data add valuable safety information on opioid therapy in the treatment of CNCP.

\section{Acknowledgments}

This work represents research conducted as part of Liu Li's doctoral dissertation in the Department of Epidemiology at the Boston University School of Public Health. We thank Drs. James Kaye and Tuhina Neogi for their critical review. We thank all the general practitioners who contribute information to the GPRD for their continuing effort and cooperation.

\section{References}

1. Chou R, Clark E, Helfand M. Comparative efficacy and safety of long-acting oral opioids for chronic non-cancer pain: A systematic review. J Pain Symptom Manage 2003; 26:1026-1048.

2. Chou R, Fanciullo GJ, Fine PG, Adler JA, Ballantyne JC, Davies P, Donovan MI, Fishbain DA, Foley KM, Fudin J, Gilson AM, Kelter A, Mauskop A, O'Connor PG, Passik SD, Pasternak GW, Portenoy RK, Rich BA, Roberts RG, Todd KH, Miaskowski C; American Pain Society-American Academy of Pain Medicine Opioids Guidelines Panel. Clinical guidelines for the use of chronic opioid therapy in chronic noncancer pain. J Pain 2009; 10:113-130.

3. Gallagher RM, Rosenthal LJ. Chronic pain and opiates: Balancing pain control and risks in long-term opioid treatment. Arch Phys Med Rehabil 2008; 89:S77-S82.

4. Pergolizzi J, Böger RH, Budd K, Dahan A, Erdine S, Hans G, Kress HG, Langford R, Likar R, Raffa RB, Sacerdote P. Opioids and the management of chronic severe pain in the elderly: Consensus statement of an International Expert Panel with focus on the six clinically most often used World Health Organization Step III opioids (buprenorphine, fentanyl, hydromorphone, methadone, morphine, oxycodone). Pain Pract 2008; 8:287-313.

5. Trescot AM, Glaser SE, Hansen H, Benyamin R, Patel S, Manchikanti L. Effectiveness of opioids in the treatment of chronic non-cancer pain. Pain Physician 2008; 11:S181-S200.

6. Trescot AM, Helm S, Hansen $H$, Benyamin R, Glaser SE, Adlaka R, Patel S, Manchikanti L. Opioids in the management of chronic non-cancer pain: An update of American Society of the Interventional Pain Physicians' (ASIPP) Guidelines. Pain Physician 2008; 11:S5-S62.

7. Moore RA, McQuay HJ. Prevalence of opioid adverse events in chronic nonmalignant pain: Systematic review of randomised trials of oral opioids. Arthritis Res Ther 2005; 7:R1046-R1051.

8. Benyamin R, Trescot AM, Datta S, Buenaventura R, Adlaka R, Sehgal N, Glaser $\mathrm{SE}$, Vallejo R. Opioid complications and side effects. Pain Physician 2008; 11:S105Si2o.

9. U.S. Food and Drug Administration, MedWatch: The FDA Safety Information and Adverse Event Reporting Program. Propoxyphene: Withdrawal - risk of cardiac toxicity. Available at: www.fda.gov/ Safety/MedWatch/Safetylnformation/ SafetyAlertsforHumanMedicalProducts/ ucm234389.htm; accessed 05/10/2011.

10. Azizi F, Vagenakis AG, Longcope C, Ingbar SH, Braverman LE. Decreased serum testosterone concentration in male heroin and methadone addicts. Steroids 1973; 22:467-472.

11. Cushman P, Jr. Plasma testosterone in narcotic addiction. Am J Med 1973; 55:452458.
12. Mendelson JH, Mendelson JE, Patch VD. Plasma testosterone levels in heroin addiction and during methadone maintenance. J Pharmacol Exp Ther 1975; 192:211-217.

13. Abs R, Verhelst J, Maeyaert J, Van Buyten JP, Opsomer F, Adriaensen H, Verlooy J, Van Havenbergh T, Smet M, Van Acker K. Endocrine consequences of long-term intrathecal administration of opioids. J Clin Endocrinol Metab 2000; 85:2215-2222.

14. Daniell HW. Hypogonadism in men consuming sustained-action oral opioids. J Pain 2002; 3:377-384.

15. Finch PM, Roberts L), Price L, Hadlow NC, Pullan PT. Hypogonadism in patients treated with intrathecal morphine. Clin J Pain 2000; 16:251-254.

16. Roberts LJ, Finch PM, Pullan PT, Bhagat $\mathrm{Cl}$, Price LM. Sex hormone suppression by intrathecal opioids: A prospective study. Clin J Pain 2002; 18:144-148.

17. Daniell HW. DHEAS deficiency during consumption of sustained-action prescribed opioids: Evidence for opioid-induced inhibition of adrenal androgen production. J Pain 2006; 7:901-907.

18. Daniell HW. Opioid endocrinopathy in women consuming prescribed sustained-action opioids for control of nonmalignant pain. J Pain 2008; 9:2836.

19. Fraser LA, Morrison D, Morley-Forster P, Paul TL, Tokmakejian S, Nicholson 
LR, Bureau Y, Friedman TC, Van Uum $\mathrm{SH}$. Oral opioids for chronic non-cancer pain: Higher prevalence of hypogonadism in men than in women. Exp Clin Endocrinol Diabetes 2009; 117:38-43.

20. Wu FC, von Eckardstein A. Androgens and coronary artery disease. Endocr Rev 2003; 24:183-217.

21. Kapoor D, Aldred H, Clark S, Channer $\mathrm{KS}$, Jones TH. Clinical and biochemical assessment of hypogonadism in men with type 2 diabetes: Correlations with bioavailable testosterone and visceral adiposity. Diabetes Care 2007; 30:911917.

22. Ding EL, Song Y, Malik VS, Liu S. Sex differences of endogenous sex hormones and risk of type 2 diabetes: A systematic review and meta-analysis. JAMA 2006; 295:1288-1299.

23. Dhindsa S, Prabhakar S, Sethi M, Bandyopadhyay A, Chaudhuri A, Dandona $P$. Frequent occurrence of hypogonadotropic hypogonadism in type 2 diabetes. J Clin Endocrinol Metab 2004; 89:5462 5468.

24. Oh JY, Barrett-Connor E, Wedick NM, Wingard DL; Rancho Bernardo Study. Endogenous sex hormones and the development of type 2 diabetes in older men and women: The Rancho Bernardo study. Diabetes Care 2002; 25:55-60.

25. Stellato RK, Feldman HA, Hamdy O, Horton ES, McKinlay JB. Testosterone, sex hormone-binding globulin, and the development of type 2 diabetes in middle-aged men: Prospective results from the Massachusetts male aging study. Diabetes Care 2000; 23:490-494.

26. Haffner SM, Shaten J, Stern MP, Smith GD, Kuller L. Low levels of sex hormonebinding globulin and testosterone predict the development of non-insulindependent diabetes mellitus in men. MRFIT Research Group. Multiple Risk Factor Intervention Trial. Am J Epidemiol 1996; 143:889-897.

27. Kanaya $A M$, Herrington $D$, Vittinghoff E, Lin F, Grady D, Bittner V, Cauley JA, Barrett-Connor E; Heart and Estrogen/ Progestin Replacement Study. Glycemic effects of postmenopausal hormone therapy: The Heart and Estrogen/Progestin Replacement Study. A random- ized, double-blind, placebo-controlled trial. Ann Intern Med 2003; 138:1-9.

28. Margolis KL, Bonds DE, Rodabough RJ, Tinker L, Phillips LS, Allen C, Bassford T, Burke G, Torrens J, Howard BV; Women's Health Initiative Investigators. Effect of oestrogen plus progestin on the incidence of diabetes in postmenopausal women: Results from the Women's Health Initiative Hormone Trial. Diabetologia 2004; 47:1175-1187.

29. Colameco S, Coren JS. Opioid-induced endocrinopathy. J Am Osteopath Assoc 2009; 109:20-25.

30. Katz N, Mazer NA. The impact of opioids on the endocrine system. Clin J Pain 2009;25:170-175

31. Jick H, Jick SS, Derby LE. Validation of information recorded on general practitioner based computerised data resource in the United Kingdom. BM] 1991; 302:766-768.

32. Jick H, Terris BZ, Derby LE, Jick SS. Further validation of information recorded on a general practitioner based computerized data resource in the United Kingdom. Pharmacoepidemiol Drug Saf 1992; 1:347-349.

33. Jick SS, Kaye JA, Vasilakis-Scaramozza C, Garcia Rodríguez LA, Ruigómez A, Meier CR, Schlienger RG, Black C, Jick $\mathrm{H}$. Validity of the general practice research database. Pharmacotherapy 2003; 23:686-689.

34. Herrett E, Thomas SL, Schoonen WM, Smeeth L, Hall AJ. Validation and validity of diagnoses in the General Practice Research Database: A systematic review. $\mathrm{Br}$ J Clin Pharmacol 2010; 69:4-14.

35. Burke TA, Sturkenboom MC, OhmanStrickland PA, Wentworth CE, Rhoads GG. The effect of antihypertensive drugs and drug combinations on the incidence of new-onset type-2 diabetes mellitus. Pharmacoepidemiol Drug Saf 2007; 16:979-987.

36. Shawe J, Mulnier H, Nicholls P, Lawrenson R. Use of hormonal contraceptive methods by women with diabetes. Prim Care Diabetes 2008; 2:195-199.

37. MedlinePlus, U.S. National Library of Medicine, National Institutes of Health. Type 2 diabetes - risk factors. Avail- able at: www.nlm.nih.gov/medlineplus/ency/article/002072.htm; accessed 06/30/2011.

38. Clore JN, Thurby-Hay L. Glucocorticoidinduced hyperglycemia. Endocr Pract 2009; 15:469-474.

39. American Diabetes Association, American Psychiatric Association, American Association of Clinical Endocrinologists, North American Association for the Study of Obesity. Consensus development conference on antipsychotic drugs and obesity and diabetes. Diabetes Care 2004; 27:596-601.

40. Li C, Ford ES, Li B, Giles WH, Liu S. Association of testosterone and sex hormone-binding globulin with metabolic syndrome and insulin resistance in men. Diabetes Care 2010; 33:1618-1624.

41. Goodman-Gruen D, Barrett-Connor E. Sex differences in the association of endogenous sex hormone levels and glucose tolerance status in older men and women. Diabetes Care 2000; 23:912-918.

42. Kapoor D, Malkin CJ, Channer KS, Jones $\mathrm{TH}$. Androgens, insulin resistance and vascular disease in men. Clin Endocrinol (Oxf) 2005; 63:239-250.

43. Godsland IF. Oestrogens and insulin secretion. Diabetologia 2005; 48:2213-2220.

44. Howard AA, Hoover DR, Anastos K, Wu X, Shi Q, Strickler HD, Cole SR, Cohen $\mathrm{MH}$, Kovacs A, Augenbraun M, Latham PS, Tien PC. The effects of opiate use and hepatitis $C$ virus infection on risk of diabetes mellitus in the Women's Interagency HIV Study. J Acquir Immune Defic Syndr 2010; 54:152-159.

45. Bradbury RA, Samaras K. Antiretroviral therapy and the human immunodeficiency virus--improved survival but at what cost? Diabetes Obes Metab 2008; 10:441-450.

46. Turk DC, Swanson KS, Gatchel RJ. Predicting opioid misuse by chronic pain patients: A systematic review and literature synthesis. Clin J Pain 2008; 24:497508.

47. Newnham A, Ryan R, Khunti K, Majeed A. Prevalence of diagnosed diabetes mellitus in general practice in England and Wales, 1994 to 1998. Health Statistics Quarterly 2002; 14:5-13. 